\title{
Development of Home Care Guidelines for the Caregivers of the Patients Being Discharged with Spinal Cord Injury
}

\author{
Navdeep Kaur ${ }^{1}$ Neena Vir Singh ${ }^{1}$ Sukhpal Kaur ${ }^{1}$ \\ ${ }^{1}$ National Institute of Nursing Education, Postgraduate Institute of \\ Medical Education \& Research, Chandigarh, Punjab, India \\ 2 Department of Neurosurgery, Postgraduate Institute of Medical \\ Education \& Research, Chandigarh, Punjab, India
}

\author{
Rajesh Chabra ${ }^{2}$
}

Address for correspondence Navdeep Kaur, MSc, National Institute of Nursing Education, Postgraduate Institute of Medical Education \& Research, Chandigarh, Punjab 160012, India

(e-mail: navdeeptaurus@yahoo.in).

\begin{abstract}
Keywords

- SCl

- home care guidelines

- caregiver

Background After spinal cord injury $(\mathrm{SCl})$, victims may become partially or fully dependent on the caregivers. After getting discharge from the hospital, they are cared by their caregivers. Often patients return to hospital with serious complications owing to inadequate knowledge of the caregivers. So, it is essential to meet the informational needs of the caregiver regarding home care.

Objective The aim of this study is to develop home care guidelines for the caregivers of the patients being discharged with $\mathrm{SCl}$.

Method The study was conducted in five phases. The blueprint of the home care guidelines was prepared. Face and content validity of the home care guidelines were done using the Delphi technique. An observational checklist was prepared from the final draft of the guidelines. The guidelines were found feasible after conducting a pilot study on five caregivers. To calculate the reliability of the guidelines, 30 caregivers were taught as per developed guidelines and were observed while caring for their patients by checklist. Reliability was calculated using Cronbach $\alpha$ coefficient.

Results The checklist showed Cronbach $\alpha$ of 0.91 . Guidelines were found to be feasible and reliable. Guidelines were developed in two languages: English and Hindi. Conclusion The home care guidelines are valid and reliable. Guidelines enable caregivers to provide the best possible care to their patient at home to prevent development of complications.
\end{abstract}

\section{Introduction}

A spinal cord injury ( $\mathrm{SCl}$ ) refers to any injury to the spinal cord that is caused by trauma instead of disease. It is a serious condition resulting in severe disability or death, with survivor facing myriad of health problems and multiple complications affecting their day-to-day living. There is no definite treatment for $\mathrm{SCl}$, but one can prevent further damage to the cord with medical and surgical treatment. The rehabilitation process following an SCI typically begins in the acute care settings to make the patient's life easy and to prevent complications. ${ }^{1}$ Patients with $\mathrm{SCI}$ usually require long-term care. With a huge population overcrowding the hospitals, it becomes necessary to discharge these patients to accommodate the other patients who need emergency care. Otherwise also, shorter stay of the patient in the hospital is preferred. With this changing climate of health care delivery system, a large number of clients return to their homes quicker, however, they may be too sick to be looked after in the home settings. So, they are left to the care received

April 8, 2015

accepted

May 25, 2015

published online

December 16, 2015
DOI http://dx.doi.org/

$10.1055 / \mathrm{s}-0035-1568994$ ISSN 2277-954X. (c) 2015 Neurological Surgeons' Society
of India

License terms

c) $(1) \$$ 
by their family members. The family/caregiver may not have acquired an adequate knowledge of how to give care at home. They are not just concerned with activities of daily living, such as bathing, dressing, eating, but also in many other health care matters such as prevention of contractures and pressure sore, bladder, and bowel management. ${ }^{2}$

A prospective cohort study conducted in Australia revealed that the incidence of contracture in major joints 1 year after SCI ranges from 11 to $43 \%$. The ankle, wrist, and shoulder are most commonly affected joints. Although contracture development is preventable still incidence of occurrence of contractures is quite high. ${ }^{3}$ Another study conducted in 1990 by Kwiczala et al also revealed that families and caregivers of bedridden patients have insufficient knowledge of pressure ulcer prevention. Contribution of medical staff in education of families of patients at risk in pressure ulcer prevention is minimal, indicating the need of preparation and implementation of an educational program for bedridden patients' caregivers. ${ }^{4}$ In 2010, a study was conducted which revealed that the urinary complications remain the leading cause of morbidity and one of the cause for urinary complications is the lack of guidelines for bladder management. ${ }^{5}$ Another study found that caregivers who provide care to people with $\mathrm{SCI}$ are struggling to provide quality care to improve their patients' lives in the absence of good evidence to guide their treatment decisions. ${ }^{6}$ A recent study performed at Postgraduate Institute of Medical Education \& Research, Chandigarh, India, in 2011 on 19 patients found that major complications faced by patients with SCI after discharge from hospital were bedsores (52.6\%) followed by bowel and bladder dysfunction (47.4\%) and urinary tract infection (42.1\%). Study concluded that there is a need for intensive public education regarding $\mathrm{SCI}$, its complications, their prevention, and care. ${ }^{7}$ Experience suggested that to ensure the safest care for the patient at all times and to provide quality care at home, teaching and instructions should be given to caregivers regarding various care modalities and procedures. With this background, a methodological study was conducted with the objective to develop the home care guidelines for the caregivers of the patients being discharged with SCI. Home care guidelines are the comprehensive list of the important and relevant actions or steps which are to be performed in a specific manner. The home care guidelines are meant to assist caregivers in providing adequate, safe, and efficient care to their patients in the home setting, to improve patient's quality of life, and to prevent complications.

\section{Patients and Methods}

A methodological research approach was adopted to develop the home care guidelines for caregivers of patients being discharged with SCI. The study was performed in Neurosurgical and Orthopaedic units of Nehru Hospital, Chandigarh, India. Ethical approval for the study was obtained from the Institute Ethical Committee of PGIMER, Chandigarh, India. The study was conducted in five phases.
Phase I: Preparation preliminary draft of the home care guidelines: it was divided into the following two steps: in the first step, an exhaustive literature review was done to collect recent evidences related to home care of patient with $\mathrm{SCI}$ and the role of family members in the care of the patient. Further, a rigorous review of literature related to the prevention of complications in the patients with SCI was done. Sources of literature included both electronic databases (PubMed, Medline, and CINHAL), as well as printed material (books and journals). In the second step, various protocols and tools were analyzed and items related to home care were pooled together. Selected items were organized to generate first draft of home care guidelines. Items were categorized under eight domains (personal hygiene, care of the bowel, care of the bladder, bedsore prevention, pressure sore dressing, range of motion exercises, changing position of patient, and incentive spirometry).

Phase II: The face and content validity of the home care guidelines was done with the Delphi technique. For the procedure of the Delphi technique, a panel of experts consisting of 11 members from field of nursing (medicalsurgical nursing and community health nursing) and department of neurosurgery was formulated. The sample of panelists was heterogeneous to ensure that entire spectrum of opinion could be determined. These all panelists were experts in their field and interested to engage in the whole process of this research study. The written consent was taken from all the selected experts to participate in study. Then, first draft of home care guidelines was circulated among the selected panel of experts and they were requested to give their valuable suggestions pertaining to content for its relevance, clarity, language, organization, level of understanding, accuracy of information, item order, that is, organization and sequence of the items and wording of items. The process was continued until there was a common consensus among the panel of experts. Four Delphi rounds were done to obtain the consensus, as per expert's opinion modifications in home care guidelines were made. The fourth draft of guidelines had seven domains regarding care of the patient at home with exclusion of one domain, that is, wound dressing. An observational checklist consisting of 125 items was prepared from the fourth draft of guidelines to compute reliability of these guidelines. One score was given for each correct and zero score for each incorrect or missing step.

Phase III: A pilot study was conducted on five caregivers in Neurosurgical and Orthopaedics units of Nehru Hospital, Postgraduate Institute of Medical Education \& Research, Chandigarh, India, to assess the feasibility and practicability of home care guidelines and to pretest the home care guidelines for language clarity, appropriateness, and sequence of items. Caregivers of those patients who had paraplegia or quadriplegia after SCI were included in study. Caregivers of the patients who had injury above $C_{4}$ (vertebrae) level were excluded. Purpose of the study was explained to the caregivers and a written consent was obtained. Caregivers were taught as per developed 
guidelines. Individualized demonstrations on two domains were given to the caregivers in a day. Return demonstrations of the same domains were taken from the caregivers on the next day before demonstrating the next two domains/ procedures. Researcher took 5 days to demonstrate all the domains to the caregiver of one patient. Guidelines were found to be feasible. Guidelines were easy to understand and implement. The language of the guidelines was clear, appropriate and items were organized in a sequence. The average time taken to teach the guidelines and to observe the developed skills of the caregivers was 25 to 30 minutes per day for 4 days. So, it was feasible to train the caregivers of patients as per developed home care guidelines. No further modifications were required. The draft 4 of home care guidelines was considered as final draft.

Phase IV: The reliability of developed home care guidelines was tested in phase IV. It included implementation of the home care guidelines and reliability of the guidelines that was checked by using Cronbach $\alpha$. For this, 30 caregivers were taught as per developed guidelines and they were observed while caring for their patient by checklist. Purpose of the study was explained to the caregivers, and written consent was obtained from them. Home care guidelines consisted of seven domains of care, that is, personal hygiene, bladder care, bowel care, bedsore prevention, changing position of patient, prevention of contractures, and incentive spirometry. Individualized demonstrations on two domains were given to the caregivers in a day. Return demonstrations of the same domains were taken from caregivers on next day before demonstrating the next two domains/procedures. Researcher took 5 days to demonstrate all the domains to the caregiver of one patient.

Phase V: Home care guidelines were developed in the form of booklet in two languages English and Hindi.

\section{Results}

The data were analyzed by SPPS (version 16). The Cronbach $\alpha$ was used to find out internal consistency reliability of present checklist prepared from final draft of home care guidelines. The sample size for analysis was 30 . There were total 125 items in the checklist, and the overall Cronbach $\alpha$ coefficient of the developed checklist was 0.91, which indicates the reliability and internal consistency of checklist (ideally Cronbach $\alpha$ coefficient should be 0.7 or more than 0.70). Corrected item to total score correlation was applied on all 125 items of the checklist, 120 items in the checklist had an item score to total score correlation between 0.1 and 0.9 (-Tables 1-12), whereas 5 items in the scale had an item to total score correlation less than 0.1 showing incompatibility with the overall checklist.

To check the individual contribution of items, each item was deleted one by one to see the changes in the value of Cronbach $\alpha$ coefficient. But none of the items showed any increase in value of Cronbach $\alpha$. Rather, the value of Cronbach $\alpha$ coefficient remained same or it decreased. This indicates all items contributed to tool. Even on deleting certain items, having item to total correlation less than 0.1 , the value of Cronbach $\alpha$ did not increase. Hence, these five items also contributed to the reliability of the checklist. The average scale mean was 148.38 . When scale mean if item deleted was applied on 125 items of checklist, the scale mean if item is deleted was in the range of 148.23 to 148.50 and none of the item had shown an increase in the value of average scale mean rather it remained same or it decreased. It means checklist is internally consistent by taking all the 125 items.

\section{Discussion}

This methodological study was planned with the objective to develop the home care guidelines for the caregivers of patients being discharged with $\mathrm{SCI}$ in selected units of Nehru Hospital, Postgraduate Institute of Medical Education \& Research, Chandigarh, India. Home care guidelines are the comprehensive list of the important and relevant actions or steps which are to be performed in a specific manner. The home care guidelines are required to ensure that quality care is being provided to patients in the home setting, and to prevent complications.

Developing countries such as India do not have good number of rehabilitation centers which are necessary for patients with SCI. Moreover, available health facilities are too expensive that everyone cannot afford them. Caregivers do not have adequate knowledge to care for their patient at

Table 1 Reliability analysis of items of checklist of personal hygiene

\begin{tabular}{|l|l|l|l|}
\hline Item of checklist & $\begin{array}{l}\text { Scale mean if } \\
\text { item deleted }\end{array}$ & $\begin{array}{l}\text { Corrected item } \\
\text { total correlation }\end{array}$ & $\begin{array}{l}\text { Cronbach } \boldsymbol{\alpha} \text { if } \\
\text { item deleted }\end{array}$ \\
\hline Gives bed bath daily & 148.40 & 0.141 & 0.910 \\
\hline Gives eye care 2 times a day & 148.40 & 0.186 & 0.910 \\
\hline Gives oral care daily & 148.43 & 0.137 & 0.910 \\
\hline Maintains perineal hygiene & 148.23 & 0.194 & 0.910 \\
\hline Gives hair care as per need & 148.26 & 0.154 & 0.910 \\
\hline Gives hand and feet care as per need & 148.33 & 0.119 & 0.910 \\
\hline
\end{tabular}

Notes: overall scale mean is 148.38 ; overall reliability of the checklist is 0.91 (Cronbach $\alpha$ ). 
Table 2 Reliability analysis of items of checklist of the bladder care

\begin{tabular}{|c|c|c|c|}
\hline Item of checklist & $\begin{array}{l}\text { Scale mean } \\
\text { if item deleted }\end{array}$ & $\begin{array}{l}\text { Corrected } \\
\text { item total } \\
\text { correlation }\end{array}$ & $\begin{array}{l}\text { Cronbach } \\
\alpha \text { if item } \\
\text { deleted }\end{array}$ \\
\hline Collects equipments & 148.33 & 0.166 & 0.910 \\
\hline Cleans the genital area & 148.26 & 0.133 & 0.910 \\
\hline Takes catheter and wipes it up to $25 \mathrm{~cm}$ first with soap and wet swabs & 148.26 & 0.270 & 0.910 \\
\hline Applies jelly to catheter tip up to $15-25 \mathrm{~cm}$ & 148.50 & 0.228 & 0.910 \\
\hline Immerses other end of catheter in receptacle & 148.26 & 0.118 & 0.910 \\
\hline Holds penis at right angle to body/separates vulva with one hand & 148.40 & 0.147 & 0.910 \\
\hline Inserts lubricated catheter slowly in urethra & 148.43 & $0.030^{\mathrm{a}}$ & 0.910 \\
\hline Asks the patient to take deep breaths and advances the catheter & 148.23 & 0.881 & 0.906 \\
\hline Stops when urine begins to drain & 148.50 & 0.199 & 0.910 \\
\hline Allows urine to drain in the receptacle & 148.43 & 0.186 & 0.910 \\
\hline Pinches the catheter and removes it slowly & 148.46 & $0.003^{\mathrm{a}}$ & 0.910 \\
\hline Washes it with soap and water, hangs to dry it & 148.33 & 0.792 & 0.907 \\
\hline Discards urine after measuring and observing it & 148.30 & 0.718 & 0.907 \\
\hline Washes hands & 148.30 & 0.939 & 0.906 \\
\hline
\end{tabular}

altems in checklist which shows item to total correlation $<0.2$.

Notes: overall scale mean is 148.38 ; Overall reliability of the checklist is 0.91 (Cronbach $\alpha$ ).

Table 3 Reliability analysis of items of checklist of the bowel care

\begin{tabular}{|l|l|l|l|}
\hline Item of checklist & $\begin{array}{l}\text { Scale mean if } \\
\text { item deleted }\end{array}$ & $\begin{array}{l}\text { Corrected item- } \\
\text { total correlation }\end{array}$ & $\begin{array}{l}\text { Cronbach } \boldsymbol{\alpha} \text { if } \\
\text { item deleted }\end{array}$ \\
\hline Collect equipments & 148.30 & 0.939 & 0.906 \\
\hline Washes hands & 148.33 & 0.792 & 0.907 \\
\hline Provides privacy & 148.33 & 0.792 & 0.907 \\
\hline Positions patient on side & 148.30 & 0.939 & 0.906 \\
\hline Places plastic sheet under buttocks & 148.33 & 0.792 & 0.907 \\
\hline Wears gloves & 148.30 & 0.939 & 0.906 \\
\hline Lubricates index finger with jelly & 148.33 & 0.792 & 0.907 \\
\hline Inserts finger into rectum slowly & 148.30 & 0.939 & 0.906 \\
\hline Loosens the fecal mass by massaging around it & 148.30 & 0.939 & 0.906 \\
\hline Brings fecal mass downward to the end of the rectum & 148.30 & 0.939 & 0.906 \\
\hline Allows the patient to rest in between & 148.33 & 0.792 & 0.907 \\
\hline Removes small sections of feces at a time and collect in bedpan & 148.30 & 0.939 & 0.906 \\
\hline Washes the anal area and buttocks & 148.30 & 0.939 & 0.906 \\
\hline
\end{tabular}

Notes: Overall scale mean is 148.38 ; overall reliability of the checklist is 0.91 (Cronbach $\alpha$ ).

home. Hence, the researcher through this study attempted to devise home care guidelines to ensure that caregivers have sufficient knowledge and skills to care for their patient in home settings that required to improve quality of patient care and to prevent complications.

Studies revealed that complications in patients were less in those to whom teaching was given on clean intermittent catheterization, bowel program, position the patient correctly and to eliminate pressure in the injured area, inspection of the skin, and hygiene. ${ }^{8,9}$ So, in this study, the researcher focused her research around the care of a bedridden patient and developed the "home care guidelines for the caregivers of the patients being discharged with spinal cord injury."

In this study, "home care guidelines for the caregivers of the patients being discharged with spinal cord injury" were developed in five phases. Phase I was preliminary preparation of the "Homecare guidelines." In phase II, the 
152 Home Care Guidelines for Caregivers of Patient with Spinal Cord Injury Kaur et al.

Table 4 Reliability analysis of items of checklist on prevention of the bedsore

\begin{tabular}{|c|c|c|c|}
\hline Item of checklist & $\begin{array}{l}\text { Scale mean } \\
\text { if item deleted }\end{array}$ & $\begin{array}{l}\text { Corrected } \\
\text { item-total } \\
\text { correlation }\end{array}$ & $\begin{array}{l}\text { Cronbach } \\
\alpha \text { if item } \\
\text { deleted }\end{array}$ \\
\hline Keeps bedsheet wrinkle free & 148.33 & 0.881 & 0.906 \\
\hline Keeps bedsheet dry & 148.43 & 0.590 & 0.908 \\
\hline Changes the position of the patient every $2 \mathrm{~h}$ & 148.43 & 0.333 & 0.909 \\
\hline Uses comfort devices for the patient & 148.46 & 0.477 & 0.909 \\
\hline Cleans back from sacrum toward shoulder in circular motions two times a day & 148.36 & 0.591 & 0.908 \\
\hline
\end{tabular}

Notes: overall scale mean is 148.38; Overall reliability of the checklist is 0.91 (Cronbach $\alpha$ ).

Table 5 Reliability analysis of items of checklist for moving the patient up in bed toward head end

\begin{tabular}{|l|l|l|l|}
\hline Item of checklist & $\begin{array}{l}\text { Scale mean if } \\
\text { item deleted }\end{array}$ & $\begin{array}{l}\text { Corrected item- } \\
\text { total correlation }\end{array}$ & $\begin{array}{l}\text { Cronbach } \alpha \\
\text { if item deleted }\end{array}$ \\
\hline Makes the bed flat & 148.33 & 0.264 & 0.910 \\
\hline Removes the pillow from under the head & 148.36 & 0.480 & 0.909 \\
\hline 2-3 caregivers stand on the right side of bed & 148.33 & 0.379 & 0.909 \\
\hline $\begin{array}{l}\text { First caregiver assumes the responsibility for supporting } \\
\text { the head, shoulder, and chest }\end{array}$ & 148.40 & 0.179 & 0.910 \\
\hline Second caregiver supports the hips & 148.46 & 0.152 & 0.910 \\
\hline Third caregiver holds the knees from underside & 148.40 & 0.113 & 0.910 \\
\hline One caregiver gives the signal by counting 1, 2, 3 & 148.30 & 0.606 & 0.908 \\
\hline $\begin{array}{l}\text { All the caregivers move the patient up and toward } \\
\text { the center of bed at the count of 3 }\end{array}$ & 148.30 & .606 & 0.908 \\
\hline Desired position is given to the patient & 148.40 & 0.681 & 0.908 \\
\hline Places the pillows under the head of the patient or wherever desired & 148.30 & 0.939 & 0.906 \\
\hline
\end{tabular}

Notes: Overall scale mean is 148.38; overall reliability of the checklist is 0.91 (Cronbach $\alpha$ ).

Table 6 Reliability analysis of items of checklist for moving the patient up in bed with bedsheet

\begin{tabular}{|l|l|l|l|}
\hline Item of checklist & $\begin{array}{l}\text { Scale mean if } \\
\text { item deleted }\end{array}$ & $\begin{array}{l}\text { Corrected item- } \\
\text { total correlation }\end{array}$ & $\begin{array}{l}\text { Cronbach } \alpha \text { if } \\
\text { item deleted }\end{array}$ \\
\hline $\begin{array}{l}\text { Places the sheet under the patient extending } \\
\text { from shoulder to thigh }\end{array}$ & 148.36 & 0.325 & 0.910 \\
\hline Two caregivers stand on each side of bed & 148.43 & 0.269 & 0.910 \\
\hline Grasps the sheet firmly near the patient & 148.43 & 0.272 & 0.910 \\
\hline $\begin{array}{l}\text { Pulls the sheet along with the patient till } \\
\text { desired position is reached }\end{array}$ & 148.46 & 0.252 & 0.910 \\
\hline Makes the patient comfortable & 148.33 & 0.264 & 0.910 \\
\hline
\end{tabular}

Notes: Overall scale mean is 148.38; overall reliability of the checklist is 0.91 (Cronbach $\alpha$ ).

content and face validity was done with the Delphi technique. Delphi technique has also been used by Kirkwood et $\mathrm{al}^{10}$ to determine nursing research priorities in the North Glasgow University Hospitals. They found that three Delphi rounds were sufficient to reach consensus. In this study, modifications were made after three Delphi rounds, although fourth round was also taken to reach the final consensus. As there was common consensus among panelists, therefore no further modifications were required to finalize draft of "home care guidelines for the caregivers of the patients being discharged with spinal cord injury."

In phase III, feasibility was checked by conducting a pilot study. The pilot study was conducted in the wards of orthopedic and neurosurgical departments. In phase IV, the internal consistency (reliability) of the "home care guidelines" was checked by Cronbach $\alpha$ by using SPSS version 16. The Cronbach $\alpha$ coefficient value of the checklist prepared from developed home care guidelines was 
Table 7 Reliability analysis of items of checklist for moving the patient from back to side-lying position

\begin{tabular}{|c|c|c|c|}
\hline Item of checklist & $\begin{array}{l}\text { Scale mean } \\
\text { if item } \\
\text { deleted }\end{array}$ & $\begin{array}{l}\text { Corrected } \\
\text { item-total } \\
\text { correlation }\end{array}$ & $\begin{array}{l}\text { Cronbach } \\
\alpha \text { if item } \\
\text { deleted }\end{array}$ \\
\hline Brings patient toward the right side of bed & 148.30 & 0.522 & 0.908 \\
\hline $\begin{array}{l}\text { Keeps the arm of the patient away from caregiver along the side } \\
\text { of head, face and arm }\end{array}$ & 148.36 & 0.714 & 0.907 \\
\hline Keeps the arm which is near to the caregiver, across the chest of the patient & 148.46 & 0.263 & 0.910 \\
\hline Flexs near leg over the farthest leg of patient & 148.46 & 0.271 & 0.910 \\
\hline Places his/her arms under the shoulder, hips & 148.33 & 0.385 & 0.909 \\
\hline Rolls the patient gently away from self to put him/her in side-lying position & 148.43 & $0.023^{\mathrm{a}}$ & 0.910 \\
\hline Places one pillow in between knees, under the back, arm head & 148.30 & 0.428 & 0.909 \\
\hline Makes the patient comfortable & 148.50 & 0.181 & 0.910 \\
\hline
\end{tabular}

altems in checklist which shows item to total correlation $<0.2$.

Notes: Overall scale mean is 148.38 . Overall reliability of the checklist is 0.91 (Cronbach $\alpha$ ).

Table 8 Reliability analysis of items of checklist for moving the patient from bed to trolley

\begin{tabular}{|l|l|l|l|}
\hline Item of checklist & $\begin{array}{l}\text { Scale mean if } \\
\text { item deleted }\end{array}$ & $\begin{array}{l}\text { Corrected item- } \\
\text { total correlation }\end{array}$ & $\begin{array}{l}\text { Cronbach } \alpha \\
\text { if item deleted }\end{array}$ \\
\hline $\begin{array}{l}\text { 2-3 caregivers stand on the same side of the bed } \\
\text { to which they want to turn the patient }\end{array}$ & 148.30 & 0.351 \\
\hline Places the client's arms across the chest & 148.46 & 0.239 & 0.910 \\
\hline Leans onto his/her trunk and flexs his/her hips, knee, and ankles & 148.33 & 0.863 & 0.906 \\
\hline All caregivers reach over the patient & 148.30 & 0.939 & 0.906 \\
\hline First caregiver assumes the responsibility for supporting the shoulders & 148.50 & 0.142 & 0.910 \\
\hline Second caregiver supports the hips & 148.43 & 0.144 & 0.910 \\
\hline Third caregiver holds the knees of patient & 148.40 & 0.189 & 0.910 \\
\hline All the caregivers roll the patient to lateral position at the count of 3 & 148.36 & 0.554 & 0.908 \\
\hline $\begin{array}{l}\text { Supports the patient's head, back, and upper and } \\
\text { lower extremities with pillows }\end{array}$ & 148.33 & 0.173 & 0.910 \\
\hline Makes the patient comfortable & 148.33 & 0.236 & 0.910 \\
\hline
\end{tabular}

Notes: Overall scale mean is 148.38; Overall reliability of the checklist is 0.91 (Cronbach $\alpha$ ).

established at 0.91, providing the reliability of home care guidelines. In a similar methodological study by Kausal et $\mathrm{al}^{11}$ on, "development of nursing checklist to receive patients in ICU," the value of Cronbach $\alpha$ was 0.97 .

Katz et $\mathrm{al}^{12}$ reported similar findings on the internal consistency of "The Dynamic Occupational Therapy Cognitive Assessment for Children." The reported Cronbach $\alpha$ was 0.77 . Benson and Koomar ${ }^{13}$ also reported almost same findings. They assessed the internal consistency of "gravitational insecurity" outcome measure. The $\alpha$ of the total test score was 0.71 .

Similar findings were reported on the internal consistency of the "audit tool." An "audit tool" was developed for auditing the family records in a methodological study. Internal consistency of the tool was checked with Cronbach $\alpha$ coefficient, and the value was 0.73 which indicated as the reliability of the tool. ${ }^{14}$
Another study on the "development of birth preparedness tool" 15 reported the similar findings of the Cronbach $\alpha$ coefficient with value of 0.81 , which determined the reliability of the tool.

In the phase $\mathrm{V}$ of this study, home care guidelines were developed in the form of booklet in two languages: English and Hindi.

In this study, valid and reliable home care guidelines were developed for the caregivers of patients being discharged with $\mathrm{SCI}$. These home care guidelines were developed and prepared in the form of booklet in two languages: English and Hindi.

\section{Conclusion}

The complications that arise once the patient with $\mathrm{SCI}$ is discharged from hospital because of insufficient knowledge of caregiver's presents unique challenges for the health care 
154 Home Care Guidelines for Caregivers of Patient with Spinal Cord Injury Kaur et al.

Table 9 Reliability analysis of items of checklist for exercises of the shoulder, elbow, and forearm

\begin{tabular}{|l|l|l|l|}
\hline Item of checklist & $\begin{array}{l}\text { Scale mean if } \\
\text { item deleted }\end{array}$ & $\begin{array}{l}\text { Corrected item- } \\
\text { total correlation }\end{array}$ & $\begin{array}{l}\text { Cronbach } \alpha \\
\text { if item deleted }\end{array}$ \\
\hline Makes the patient comfortable in bed & 148.433 & 0.290 & 0.910 \\
\hline $\begin{array}{l}\text { Raises the right arm above the head up to maximum possible } \\
\text { height and then brings it back (sitting position) }\end{array}$ & 148.36 & 0.268 \\
\hline $\begin{array}{l}\text { Raises the right arm to the side of the body and then above the } \\
\text { head with palm away from the head and brings the arm back }\end{array}$ & 148.33 & 0.333 & 0.909 \\
\hline Moves the right arm in full circle vertically & 148.36 & 0.190 & 0.910 \\
\hline Brings the shoulder toward the body & 148.43 & 0.234 & 0.910 \\
\hline Take the shoulder away from the body & 148.46 & 0.198 & 0.910 \\
\hline Performs all exercises on left side of body & 148.33 & 0.037 & 0.910 \\
\hline Bends the elbows and moves the lower arm toward its shoulder joint & 148.36 & 0.365 & 0.909 \\
\hline Straightens the elbow by lowering the lower arm again & 148.40 & 0.303 & 0.910 \\
\hline Performs all exercises on other side of body & 148.33 & 0.179 & 0.910 \\
\hline $\begin{array}{l}\text { Keeps elbow along the side of the body and lower arm at 90 degree } \\
\text { to the elbow in horizontal position }\end{array}$ & 148.46 & 0.153 & 0.910 \\
\hline Rotates the forearm so that the palm now faces down units & 148.30 & 0.127 & 0.910 \\
\hline Performs same exercises on the other arm & 148.46 & 0.232 & 0.910 \\
\hline
\end{tabular}

Notes: Overall scale mean is 148.38; Overall reliability of the checklist is 0.91 (Cronbach $\alpha$ ).

Table 10 Reliability analysis of items of checklist for exercises of the wrist, fingers, and thumb

\begin{tabular}{|c|c|c|c|}
\hline Item of checklist & $\begin{array}{l}\text { Scale mean if } \\
\text { item deleted }\end{array}$ & $\begin{array}{l}\text { Corrected item- } \\
\text { total correlation }\end{array}$ & $\begin{array}{l}\text { Cronbach } \alpha \\
\text { if item deleted }\end{array}$ \\
\hline Straightens the wrist. Palm should be facing down units & 148.26 & 0.107 & 0.910 \\
\hline Bends the wrist upward and backward & 148.26 & 0.034 & 0.910 \\
\hline Bends the wrist forward and downward & 148.33 & 0.162 & 0.910 \\
\hline Twists wrist to the right and then to left & 148.26 & 0.186 & 0.910 \\
\hline Repeat same exercises on other hand & 148.46 & 0.224 & 0.910 \\
\hline Makes a fist and tightens the fist & 148.50 & 0.114 & 0.910 \\
\hline Relaxes and opens the fist & 148.40 & $0.083^{\mathrm{a}}$ & 0.910 \\
\hline Straightens and stretches the fist & 148.400 & 0.096 & 0.910 \\
\hline Bends fingers back toward the arm as far as possible & 148.36 & $0.007^{\mathrm{a}}$ & 0.910 \\
\hline Spreads fingers apart and brings fingers together & 148.36 & 0.094 & 0.910 \\
\hline Places squeezing ball in hand and presses it & 148.33 & 0.259 & 0.910 \\
\hline Performs all exercises on other hand & 148.33 & 0.190 & 0.910 \\
\hline $\begin{array}{l}\text { Bends the thumb toward the center of the palm and } \\
\text { then back to its original position }\end{array}$ & 148.40 & 0.121 & 0.906 \\
\hline Touches thumb to each finger of same hand & 148.33 & 0.173 & 0.910 \\
\hline Extends thumb laterally away from the index finger & 148.43 & 0.193 & 0.910 \\
\hline Moves thumb back toward hand & 148.40 & 0.276 & 0.906 \\
\hline Repeats exercises on the other hand & 148.26 & 0.175 & 0.910 \\
\hline
\end{tabular}

altems in checklist which shows item to total correlation $<0.2$.

Notes: Overall scale mean is 148.38; Overall reliability of the checklist is 0.91 (Cronbach $\alpha$ ). 
Table 11 Reliability analysis of items of checklist for exercises of the leg, knee, ankle, and toes

\begin{tabular}{|l|l|l|l|}
\hline Item of checklist & $\begin{array}{l}\text { Scale mean if } \\
\text { item deleted }\end{array}$ & $\begin{array}{l}\text { Corrected item- } \\
\text { total correlation }\end{array}$ & $\begin{array}{l}\text { Cronbach } \alpha \\
\text { if item deleted }\end{array}$ \\
\hline Moves the straighten leg up as far as possible and bring it down & 148.43 & 0.112 & 0.909 \\
\hline $\begin{array}{l}\text { Moves the straighten leg away from the body laterally as far as } \\
\text { possible and then back toward the midline }\end{array}$ & 148.26 & 0.129 & 0.910 \\
\hline Turns the leg toward the other leg and then back & 148.30 & 0.231 & 0.907 \\
\hline Repeats all the exercises on the other leg & 148.36 & 0.115 & 0.910 \\
\hline Makes the patient to lie in bed comfortably & 148.26 & 0.139 & 0.910 \\
\hline Bends the knee and then straighten it again & 148.33 & 0.100 & 0.910 \\
\hline Repeats on the other knee & 148.50 & 0.246 & 0.906 \\
\hline Moves foot so that toes are pointed upunits & 148.50 & 0.176 & 0.910 \\
\hline Moves foot so that toes are pointed downward & 148.36 & 0.164 & 0.910 \\
\hline Turns the ankles to the left and then right & 148.46 & 0.136 & 0.910 \\
\hline Repeats all these exercises on other side & 148.26 & 0.118 & 0.910 \\
\hline Spreads toes apart & 148.50 & 0.190 & 0.909 \\
\hline Curls toes downward & 148.33 & 0.179 & 0.906 \\
\hline Straightens toes and brings toes together again & 148.30 & 0.192 & 0.910 \\
\hline Performs all exercises on other toe & 148.50 & 0.209 & 0.910 \\
\hline
\end{tabular}

Notes: Overall scale mean is 148.38; Overall reliability of the checklist is 0.91 (Cronbach $\alpha$ ).

Table 12 Reliability analysis of items of checklist for incentive spirometry

\begin{tabular}{|l|l|l|l|}
\hline Item of checklist & $\begin{array}{l}\text { Scale mean if } \\
\text { item deleted }\end{array}$ & $\begin{array}{l}\text { Corrected } \\
\text { item-total } \\
\text { correlation }\end{array}$ & $\begin{array}{l}\text { Cronbach } \alpha \\
\text { if item deleted }\end{array}$ \\
\hline $\begin{array}{l}\text { Gives sitting position (paraplegic patient) or raise the head end of bed } \\
\text { with help of pillows (quadriplegia) }\end{array}$ & 148.50 & 0.133 & 0.910 \\
\hline $\begin{array}{l}\text { Asks the patient to hold spirometer in an upright position } \\
\text { (paraplegic patients) or holds spirometer in his/her hands } \\
\text { (quadriplegic patients) }\end{array}$ & 148.50 & 0.228 & 0.910 \\
\hline $\begin{array}{l}\text { Asks the patient to place the mouthpiece in his/her mouth and } \\
\text { seal his/her lips tightly around it }\end{array}$ & 148.33 & 0.150 & 0.910 \\
\hline Asks the patient to breath slowly and as deeply as possible & 148.33 & 0.166 & 0.910 \\
\hline Asks the patient to raise the ball toward the top of the column & 148.40 & 0.263 & 0.910 \\
\hline Asks the patient to hold his/her breath as long as possible & 148.36 & 0.184 & 0.910 \\
\hline Asks the patient to allow the ball to fall to the bottom of the column & 148.40 & 0.164 & 0.910 \\
\hline Asks the patient to rest for a few seconds & 148.33 & 0.396 & 0.909 \\
\hline $\begin{array}{l}\text { Asks the patient to repeat same for at least 10 times every } \\
\text { hour when he/she is awake }\end{array}$ & 148.43 & 0.193 & 0.911 \\
\hline
\end{tabular}

Notes: Overall scale mean is 148.38; Overall reliability of the checklist is 0.91 (Cronbach $\alpha$ ).

professionals. The results indicate that "home care guidelines for the caregiver of the patients being discharged with SCl" are valid and reliable and can be applied in daily practice. These guidelines address interventions tailored to enable caregivers to provide best possible care to their patient at home to prevent the development of complications.

\section{References}

1 Hicky JV. The Clinical Practice of Neurology and Neurological Nursing. Philadelphia, PA: J. B. Lippincott; 2005

2 Johnson EA, Jackson JE. Teaching the home care client. Nurs Clin North Am 1989;24(3):687-693

3 Diong J, Harvey LA, Kwah LK, et al. Incidence and predictors of contracture after spinal cord injury-a prospective cohort study. Spinal Cord 2012;50(8):579-584 
4 Kwiczala-Szydłowska S, Skalska A, Grodzicki T. Pressure ulcer prevention-evaluation of awareness in families of patients at risk. Przegl Lek 2005;62(12):1393-1397

5 Eves FJ, Rivera N. Prevention of urinary tract infections in persons with spinal cord injury in home health care. Home Healthc Nurse 2010;28(4):230-241

6 Guihan M, Goldstein B, Smith BM, Schwartz A, Manheim LM. SCI health care provider attitudes about pressure ulcer management. J Spinal Cord Med 2003;26(2):129-134

7 Kaur J, Kaur S, Chabra R. Functional dependence and impairment in patients with spinal trauma and associated complications. Indian Journal of Continuing Nursing Education 2014;15(1): 93-97

8 Afsar SI, Yemisci OU, Cosar SN, Cetin N. Compliance with clean intermittent catheterization in spinal cord injury patients: a long-term follow-up study. Spinal Cord 2013; 51(8):645-649

9 Engkasan JP, Sudin SS. Neurogenic bowel management after spinal cord injury: Malaysian experience. J Rehabil Med 2013; 45(2):141-144
10 Kirkwood M, Wales A, Wilson A. Delphi study to determine nursing research priporities in North Glasgow university hospitals. Int J Nurs Stud 2005;43:560-566

11 Kaushal RK, Kapoor S, Kaur S, Bhagat H. A methodological study to develop a "Nursing Checklist" for receiving patients in ICUs. Nursing and Midwifery Research Journal 2015;11(1):1-11

12 Katz N, Golstand S, Bar-Ilan RT, Parush S. The Dynamic Occupational Therapy Cognitive Assessment for Children (DOTCA-Ch): a new instrument for assessing learning potential. Am J Occup Ther 2007;61(1):41-52

13 May-Benson TA, Koomar JA. Identifying gravitational insecurity in children: A pilot study. American Journal of Occupational Therapy 2007;61:142-147

14 Bandana WI, Saini SK. Development of audit tool: A methodological study for auditingthe family health records. Nursing and Midwifery Research Journal 2009;5(4):166-175

15 Kaur V, Saini SK, Walia I. Development of birth preparedness tool- A tool to assess mother preparedness for delivery, postnatal and new born care. Nursing and Midwifery Research Journal 2009;5:45-58 